\title{
Intermittent self catheterisation with hydrophilic, gel reservoir, and non-coated catheters: a systematic review and cost effectiveness analysis
}

\author{
OPEN ACCESS
}

\author{
Sarah L Bermingham senior health economist ${ }^{1}$, Sarah Hodgkinson senior research fellow and \\ project manager ${ }^{1}$, Sue Wright team leader infection prevention and control ${ }^{2}$, Ellie Hayter professional \\ lead nursing ${ }^{3}$, Julian Spinks general practitioner ${ }^{4}$, Carol Pellowe senior lecturer infection control ${ }^{5}$ \\ ${ }^{1}$ Royal College of Physicians, National Clinical Guideline Centre, London NW1 4LE, UK; ${ }^{2}$ Peninsula Community Health, Sedgemoor Centre, St \\ Austell PL25 5AS, UK; ${ }^{3}$ Sussex Community NHS Trust, Horsham Hospital, Horsham RH12 2DR, UK; ${ }^{4}$ Court View Surgery, Strood ME2 2HA, UK; \\ ${ }^{5}$ Department of Adult Nursing, Florence Nightingale School of Nursing and Midwifery, King's College London, London SE1 8WA
}

\begin{abstract}
Objective To determine the most effective and cost effective type of catheter for patients performing intermittent self catheterisation in the community.

Design Systematic review and meta-analysis. Results were incorporated into a probabilistic Markov model to compare lifetime costs and quality adjusted life years (QALYs).

Data sources We searched Medline, Embase, and Cochrane and Cinahl databases from 2002 to 18 April 2011 to identify studies comparing hydrophilic, gel reservoir, and non-coated intermittent catheters. Earlier guidelines were used to identify papers published before 2002. To capture studies comparing clean and sterile non-coated intermittent self catheterisation, each database was searched from its date of inception to 18 April 2011.
\end{abstract}

Main outcome measures Clinical outcomes included symptomatic urinary tract infection (UTI), bacteraemia, mortality, patient preference or comfort, and number of catheters used. The economic model included downstream complications of UTI and cost effectiveness was calculated as incremental cost per QALY gained.

Results Eight studies were included in the systematic review. Most were conducted in patients with spinal cord injuries, and most of the included patients were men. People using gel reservoir and hydrophilic catheters were significantly less likely to report one or more UTIs compared with sterile non-coated catheters (absolute effect for gel reservoir $=149$ fewer per 1000 (95\% confidence interval -7 to 198), $P=0.04$; absolute effect for hydrophilic = 153 fewer per 1000 (-8 to 268), $\mathrm{P}=0.04)$. However, there was no difference between hydrophilic and sterile non-coated catheters when outcomes were measured as mean monthly UTIs (mean difference $=0.01(-0.11$ to 0.09$), P=0.84)$ or total UTIs at 1 year (mean difference $=0.18(-0.50$ to 0.86$), P=0.60)$. There was little difference in the incidence of one or more UTIs for people using clean versus sterile non-coated catheters (absolute effect $=12$ fewer per 1000 (-134 to 146), $P=0.86)$. Although the most effective, gel reservoir catheters cost $>£ 54$ 350 per QALY gained and are therefore not cost effective compared with clean non-coated self catheterisation.

Conclusion The type of catheter used for intermittent self catheterisation seems to make little difference to the risk of symptomatic UTI. Given large differences in resource use, clean non-coated catheters are most cost effective. However, because of limitations and gaps in the evidence base and the designation of non-coated catheters as single use devices, we recommend a precautionary principle should be adopted and that patients should be offered a choice between hydrophilic and gel reservoir catheters.

\section{Introduction}

Catheter associated urinary tract infection is the most common healthcare acquired infection in the world, accounting for $20-45 \%$ of all nosocomial infections. ${ }^{1}$ While most urinary tract infections (UTIs) are mild and easily resolved with appropriate antibiotic treatment, more severe infections can be devastating, resulting in bacteraemia, sepsis, and death. Due to the frequency with which they occur, they also impose a substantial economic burden on the NHS. ${ }^{2}$

The most important risk factor for the development of catheter associated UTI is the prolonged use of an indwelling catheter. 
In the 1970s, intermittent self catheterisation was introduced as the preferred method of catheterisation for patients with urinary retention. ${ }^{3}$ The most common indications for intermittent catheterisation are neurogenic bladder (caused by spinal cord injury, stroke, Parkinson's disease, multiple sclerosis, or diabetic neuropathy, for example) and refractory bladder (caused by urethral obstruction due to infection, metastases, or congenital abnormalities), but it may also be indicated in people with prostatic enlargement or who have undergone procedures or are taking medication that affects the contractility of the detrusor muscle. Intermittent self catheterisation is meant to reduce catheter associated UTIs and promote greater independence among people who have bladder emptying problems. Nevertheless, UTI remains the most common and serious complication of intermittent self catheterisation. ${ }^{5}$

Several different catheter materials and methods are available for intermittent self catheterisation. Patients may use catheters with a hydrophilic polymer surface coating, packaged with water based lubricant (gel reservoir), or non-coated. Because of the nature of their coatings, hydrophilic and gel reservoir catheters must always be discarded after each use. Non-coated catheters may either be discarded after use or washed and re-used for up to one week; these two methods are commonly referred to as sterile non-coated catheterisation and clean non-coated catheterisation, respectively. Although clean non-coated catheterisation used to be the most common method of intermittent self catheterisation on both sides of the Atlantic, ${ }^{6}$ it has recently fallen out of favour in preference for hydrophilic and gel reservoir catheters. Which material and method constitute the best approach remains uncertain. ${ }^{7-10}$ The aim of this study was to determine the most clinically effective and cost effective approach for patients performing intermittent self catheterisation in the community. Our systematic review and economic analysis were conducted as part of the 2012 update of the National Institute for Health and Clinical Excellence (NICE) guideline Infection: Prevention and Control of Healthcare-associated Infections in Primary and Community Care. $^{11}$

\section{Methods}

\section{Systematic review of clinical literature Search strategy}

A systematic literature search was conducted to identify all randomised controlled trials of intermittent self catheterisation in the published literature. Two separate searches were performed: one to identify studies comparing hydrophilic, gel reservoir, and non-coated catheters and the other to capture studies comparing clean and sterile non-coated intermittent self catheterisation. The first search was run in Medline (OVID), Embase (OVID), the Cochrane Library, and Cinahl (EBSCO) from 2002 to 18 April 2011. The previous NICE guideline was used to identify papers published before 2002. The second search was not undertaken for the 2003 guideline and was therefore conducted from the date of inception for each database to 18 April 2011. Details of the search strategy used for each database are available in appendix 1 on bmj.com as part of our supplementary information.

\section{Assessment of eligibility}

Only randomised controlled trials and randomised crossover trials of long term (>28 days) intermittent self catheterisation in community or primary care settings were included. Outcomes considered relevant for clinical decision making included symptomatic UTI, bacteraemia, mortality, patient preference or comfort, and the number of catheters used per day or week. The presence of blood in the urine and urine $\mathrm{pH}$ changes were also included. All laboratory studies, abstracts, posters, reviews, letters or editorials, foreign language publications, and unpublished studies were excluded. Asymptomatic bacteriuria was not included as an outcome because it has little clinical impact, and treatment is not recommended. ${ }^{12}$

\section{Study selection and data extraction}

Potentially relevant studies were retrieved based on title and abstract sifting. Retrieved papers were reviewed to identify studies conducted in relevant populations that reported outcomes of interest (protocols are available in appendix 2 on bmj.com and in appendix E of the full NICE guideline ${ }^{13}$ ). Studies were critically appraised by SH using the NICE Guideline Manual checklist for of risk of bias (selection, performance, attrition, and detection bias). ${ }^{14}$ The results of studies meeting inclusion criteria were pooled according to outcome, and the quality of evidence for each outcome was evaluated using the GRADE (Grading of Recommendations Assessment, Development and Evaluation) system GRADEpro software developed by the GRADE working group. ${ }^{15}$ In this system, the quality of evidence is assessed for each relevant outcome and is based on study design, limitations, and consistency and directness of the evidence, which informs the confidence the group has in the evidence presented.

\section{Data synthesis}

Where appropriate, meta-analyses were conducted to combine the results of studies using Review Manager (RevMan5) software. ${ }^{16}$ Binary outcomes were pooled using a fixed effect (Mantel-Haenszel) model. Continuous outcomes were analysed using an inverse variance method for pooling weighted mean differences; where studies had different scales, standardised mean differences were used. Statistical heterogeneity was assessed by considering the $\chi^{2}$ test for significance at $\mathrm{P}<0.1$ or an $\mathrm{I}^{2}$ statistic of $>50 \%$ to indicate significant heterogeneity. Assessments of potential differences in effect between subgroups were based on the $\chi^{2}$ tests for heterogeneity statistics between prespecified subgroups.

\section{Cost effectiveness analysis}

The results of the systematic review were incorporated into a probabilistic Markov model ${ }^{17}$ to establish the most cost effective method of intermittent self catheterisation from the perspectives of the UK National Health Service and personal social services. Results were expressed in terms of costs, quality adjusted life years (QALYs), and incremental cost per QALY gained. All costs are reported in UK pounds for the years 2009-10. Both costs and QALYs were discounted at the standard annual rate of $3.5 \%$. In the UK, an incremental cost effectiveness ratio of less than $£ 20000-30000$ is considered cost effective by policy makers. ${ }^{14}$ A detailed description of the methods used to inform this analysis is available in appendix $\mathrm{J}$ of the full NICE guideline. $^{13}$

\section{Population and comparators}

There are multiple causes of bladder dysfunction which affect a heterogeneous population. Because most of the clinical effectiveness studies included in our analysis were conducted in people with neurogenic bladder due to spinal cord injury, this was the population considered in the base case of our model. The hypothetical cohort had an average age of 40 years and was 
$80 \%$ male; this is the average age at injury and sex ratio of people with spinal cord injury according to the US national spinal cord injury database ${ }^{18}$ and was similar to the population included in the clinical trials. Age dependant background mortality rates were calculated based on UK life tables ${ }^{19}$ and a standardised mortality ratio of 5.41 for people with spinal cord injury who are aged $31-41$ years at time of injury. ${ }^{20}$ The model was run over the cohort's lifetime.

The model was not designed to evaluate the cost effectiveness of different types of intermittent catheters in children. None of the clinical trials which reported UTI as an outcome was conducted in children, and there are no published quality of life values for children with UTI or UTI related complications. ${ }^{21}$ In addition, symptomatic UTI in children and young people can cause progressive renal scarring which may lead to renal failure later in life. However, the most recent NICE guideline for UTI in children ${ }^{22}$ concluded that it was not possible to estimate the true risk of renal failure as a result of childhood UTI. Therefore, economic modelling was not considered a valid option in this population.

The comparators evaluated in our model included each of the five different materials and methods of intermittent self catheterisation included in the systematic review: hydrophilic coated catheters, gel reservoir coated catheters, sterile non-coated catheters discarded after each use, clean non-coated catheters changed once a day, and clean non-coated catheters changed once a week. Although clean non-coated self catheterisation is an established method of catheterisation in the community, non-coated catheters have been designated as single use items by the symbol on their packaging. There are also clinical factors which may influence whether clean non-coated self catheterisation is considered an option, such as a patient's ability to manipulate the catheter or undertake self catheterisation in settings such as the workplace or other communal spaces - where dignity, availability of facilities, or an increased risk of infection may be important considerations. In order to evaluate the optimal method of intermittent self catheterisation in situations where clean non-coated self catheterisation is not considered appropriate, the economic model was run over two scenarios: one for when clean non-coated self catheterisation is an alternative and one for when it is not.

\section{Model structure}

Figure $1 \Downarrow$ shows the key health states in the model and possible transitions between them during each one year cycle. The model was designed to capture the effect of treatment failure due to antibacterial resistant infection. Because of lack of available data about current and historical resistance rates, the complexity of forecasting antibiotic resistance trends over time and within populations, and a lack of examples on which to base methodological approaches, ${ }^{23}$ the main simplifying assumption was that the probability of antibiotic resistance does not change over time. Different rates of resistance were explored in sensitivity analysis. The model did not include a transition from first line or multidrug resistant UTI to bacteraemia because it was assumed that the risk of developing symptomatic UTI reported in the literature represents the cumulative probability of bacteraemia as a result of all symptomatic UTIs. Therefore, this probability was only applied once within the model. All individuals within the model were exposed to a background rate of mortality from all causes. The arrows indicate states which are associated with an excess risk of mortality.

\section{Transition probabilities}

The baseline risk of symptomatic UTI was based on the rate of UTI in people using sterile non-coated catheters in the studies included in the systematic review. ${ }^{23-27}$ The relative risk of one or more UTI identified in the systematic review was applied to the baseline risk of infection to calculate the annual probability of symptomatic UTI associated with each catheter.

Although empirical treatment is effective in most cases, a small proportion of people will experience persistent symptomatic infection. The probability of clinical failure after treatment for symptomatic UTI was obtained from a study of people with spinal cord injury receiving a course of ciprofloxacin as first line treatment. ${ }^{29}$ Among individuals with spinal cord injury, it is thought that repeated exposure to healthcare settings and antimicrobial agents increases the risk of infection with multidrug resistant organisms. The most common mechanism of resistance in organisms that cause UTI is the production of extended spectrum $\beta$ lactamases, which inactivate certain antibiotics. Based on studies of the prevalence of multidrug resistant UTI in people with spinal cord injury, ${ }^{30}{ }^{31}$ we estimated that just less than half of people who fail treatment have a multidrug resistant UTI; the remainder were assumed to experience treatment failure due to infections resistant to first line antibiotics. The probability of bacteraemia as a result of catheter associated UTI was obtained from a systematic review and meta-analysis by Saint $2000 .^{32}$ Mortality associated with multidrug resistant UTI and with bacteraemia were calculated from studies by Klevens et al $2008^{33}$ and Montgomerie 2011, ${ }^{34}$ respectively. Baseline transition probabilities are reported in table $1 \Downarrow$.

Long term studies have demonstrated that the incidence of urethral complications associated with intermittent self catheterisation tends to increase over time. ${ }^{5}$ Although hydrophilic catheters are associated with a lower surface friction according to some cytological studies, ${ }^{35}{ }^{36}$ no comparative clinical trials have evaluated the impact of this effect on patient outcomes. A baseline probability of developing urethral complications was applied as a background rate to all health states. This figure was derived from an observational study of patients using intermittent self catheterisation over an average period of 9.5 years. ${ }^{37}$ This value is at the upper end of estimates reported by other papers ${ }^{5}$ and was chosen to represent the possibility of developing urethral complications of any type, whether they are strictures, false passages, urethritis, or any other complication that could result from urethral trauma. It was conservatively assumed that the risk of developing urethral complications did not differ between catheters. This relative risk was then varied using extreme values to test the sensitivity of the model to this assumption.

\section{Quality of life}

A systematic search of the literature was performed to identify studies reporting quality of life in people with UTI and with UTI associated bacteraemia. The methods and results of this review have been reported elsewhere. ${ }^{21}$ Briefly, we identified two recent studies which measured the impact of UTI in people with spinal cord injury using a validated generic measure of health related quality of life. ${ }^{38-40}$ The authors of these studies were contacted for additional information. Although Haran and co-workers were unable to provide any further data, Vogel and colleagues granted us access to recent patient-level responses to the SF-12 health questionnaire collected as part of a longitudinal study of adults who sustained spinal cord injury as children and adolescents. ${ }^{40} 41$ The responses were grouped 
according to three categories: no UTI, UTI, and severe UTI requiring intravenous antibiotics or hospitalisation. The recall period for each group was one year. Using an algorithm developed by Gray et $\mathrm{al},{ }^{42}$ the data were mapped to EQ-5D values of health status for the UK population. Table $2 \Downarrow$ shows the values for quality of life assigned to each health state.

A recent Cochrane review of procedures for urethral narrowing did not find any quality of life data among patients treated for urethral strictures. ${ }^{43} \mathrm{~A}$ search of the Tufts Cost Effectiveness Analysis Registry ${ }^{44}$ also failed to identify any relevant utility weights. Given that urethral complications would probably result in substantial discomfort and stay in hospital, we assumed that the quality of life associated with this health state would be similar to that experienced by patients with multidrug resistant UTI.

\section{Costs}

A simple average of the cost of each type of intermittent catheter listed in the NHS Drug Tariff $\mathrm{f}^{45}$ was used as the point estimate, with the range informed by the most and least expensive unit cost for each type of catheter. The annual cost for patients using sterile catheters was based on an average of five catheters used per day. ${ }^{46}$ For patients using clean non-coated catheterisation, the systematic review included two studies in which non-coated catheters were changed either daily ${ }^{28}$ or weekly. ${ }^{26} \mathrm{~A}$ threshold analysis of the number of catheters used per day was performed in sensitivity analysis. All people using non-coated catheters were assumed to use a sterile sachet of lubricant each time they performed self catheterisation. Based on expert opinion, it was assumed that $5 \%$ of patients use a lidocaine based lubricant while the remainder use one that is water based. Because lubricant is applied to the catheter each time it is used, patients with sterile and multiple use non-coated catheters were assumed to consume equal amounts of lubricant. A monthly prescription dispensing fee was added to the cost of catheters and lubricant. Table $3 \Downarrow$ shows the average cost of each catheter and lubricant, as well as the associated average annual cost for each method of intermittent self catheterisation.

Treatment costs for catheter associated UTI were estimated from recommended treatment pathways for UTI in adults. ${ }^{47} 48$ Treatment of symptomatic UTI and UTI resistant to first line antibiotics was assumed to be managed in the community, whereas patients with multidrug resistant UTI, bacteraemia, or urethral complications were assumed to be treated in hospital. Costs incurred in the community were based on data from the 2010 NHS Drug Tariff ${ }^{45}$ and 2009-10 Personal and Social Services Research Unit. ${ }^{49}$ The cost of secondary care was calculated according to 2009-10 NHS reference costs. ${ }^{50}$ Table $4 \Downarrow$ provides a summary of the costs associated with treatment of each health state included the model.

\section{Uncertainty}

The model was built probabilistically using Monte Carlo simulation $^{51}$ to take account of uncertainty surrounding each input parameter. A probability distribution was defined for each parameter based on reported point estimates and standard errors. The model was run 10000 times. This number was chosen to ensure that Monte Carlo error was below 5\% of the standard error for both the incremental cost and incremental QALYs of each parameter compared with the baseline. Each time the model was run, values for each input parameter were randomly selected from their respective distributions. Mean costs and QALYs were calculated by averaging across all 10000 simulations, resulting in an estimate of the long term outcomes for people using different methods for intermittent self catheterisation.

\section{Sensitivity analyses}

People with bladder dysfunction are a highly diverse group of individuals. A similar model exploring the cost effectiveness of intermittent catheterisation in patients with bladder dysfunction not due to spinal cord injury was developed as part of the sensitivity analysis. In the absence of other data, it was assumed that the relative risk of symptomatic UTI was the same as that observed in people with spinal cord injury, and several cohort probabilities were changed to reflect the probability of antibiotic resistance and mortality in a more diverse population. (See appendices of the full NICE guideline ${ }^{13}$ for full details of the assumptions and parameters used to inform this analysis.) To test the robustness of the model, we also undertook one way and two way sensitivity analyses surrounding data and assumptions related to antimicrobial resistance, urethral complications, mortality, utility, resource use, and costs.

\section{Interpreting results}

The results of cost effectiveness analysis are presented as incremental cost effectiveness ratios (ICERs). ICERs are calculated by dividing the difference in costs associated with two alternative treatments by the difference in QALYs. Where more than two interventions are being compared, the ICER is calculated according to the following process: first, the interventions are ranked in terms of cost, from least to most expensive. If an intervention is more expensive and less effective than the preceding intervention, it is said to be "dominated" and is excluded from further analysis. ICERs are then calculated for each drug compared with the next most expensive non-dominated option by dividing the incremental cost by incremental QALY gain. If the ICER for a drug is higher than that of the next most effective strategy, then it is ruled out by "extended dominance." ICERs are recalculated excluding any drugs subject to dominance or extended dominance. When there are multiple comparators, the option with the greatest average net benefit may also be used to rank comparators.

NICE's report Social Value Judgements: Principles for the Development of NICE Guidance ${ }^{52}$ sets out the principles that NICE guideline development groups should consider when judging whether an intervention offers good value for money. In general, an intervention is considered to be cost effective if either the intervention dominates other relevant strategies (that is, it is both less costly in terms of resource use and more clinically effective compared with all the other relevant alternative strategies) or the intervention costs less than $£ 20$ 000 per QALY gained compared with the next best strategy.

\section{Results \\ Clinical effectiveness}

We identified 230 papers from the literature search, of which 55 were ordered for full appraisal. Of these, 47 papers did not meet the inclusion criteria for this review question (22 non-systematic reviews, 8 of inadequate study design, 7 hospital based or short term catheterisation, 8 comparing interventions not listed within the protocol, and 2 did not report any of the pre-specified outcomes). A total of eight studies were included in this review: five evaluated hydrophilic catheters compared with non-coated catheters, ${ }^{24} 25$ 53-55 one compared gel reservoir catheters with non-coated catheters, ${ }^{27}$ and two compared clean non-coated catheterisation with sterile non-coated 
catheterisation. ${ }^{26}{ }^{28}$ None of the studies included in the previous 2003 guideline met the inclusion criteria. Diagrams of the literature search and selection process are available in appendix 3 on bmj.com as part of the supplementary information.

The non-coated catheters were discarded after each use in Cardenas et al 2009 ${ }^{24}$; they were cleaned and reused up to five times a day, with a new catheter used each day in Vapnek et al $2003^{55}$ and Pachler et al $1999 .^{53}$ The studies by Giantonni et al $2001,{ }^{27}$ Sutherland et al $1996,{ }^{54}$ and De Ridder et al $2005^{25}$ did not provide details about the method of catheterisation, and we contacted the authors of these studies for clarification. De Ridder replied that the catheters used in the study were sterile. No reply was obtained from Giantonni or Sutherland; it was assumed that these studies also used sterile non-coated catheterisation. In the two studies comparing clean non-coated catheterisation with sterile non-coated catheterisation, patients in the clean arm replaced their catheters either weekly ${ }^{26}$ or daily. ${ }^{28}$

Most of the studies were conducted in patients with spinal cord injuries, and most of the patients included in these studies were men. None of the included studies reported bacteraemia or mortality. Table $5 \Downarrow$ summarises the patient characteristics and outcomes for each study.

\section{Symptomatic UTI}

According to the results of the included studies, there was no difference in the mean monthly number of UTIs (mean difference -0.01 (95\% confidence interval -0.11 to 0.09 ), $\mathrm{P}=0.84),{ }^{55}$ total number of UTIs at one year (mean difference 0.18 ( -0.50 to 0.86$), \mathrm{P}=0.60),{ }^{24}$ or total antibiotic treatment episodes at one year (mean difference $-0.88(-1.58$ to -0.18$)$ $\mathrm{P}=0.01)^{24}$ for people using hydrophilic coated catheters compared with those using non-coated catheters. These outcomes were of moderate quality according to GRADE criteria.

People using gel reservoir and hydrophilic catheters were significantly less likely to report one or more UTIs compared with those using sterile non-coated catheters (fig $2 \Downarrow$ ). However, the confidence intervals for these values were wide and overlapping. There was no significant difference in the incidence of symptomatic UTI for people using clean versus sterile non-coated catheters for long term intermittent self catheterisation.

\section{Patient preference}

In the study by de Ridder et al $2005^{25}$ a slightly greater proportion of patients or carers using hydrophilic catheters reported that they were very satisfied compared with patients and helpers using sterile non-coated catheters at six months (risk ratio 1.79 (95\% confidence interval 0.70 to 4.59 ), $\mathrm{P}=0.23$ ) and 12 months (risk ratio 1.38 (0.55 to 3.45$), \mathrm{P}=0.49$ ).

Using a visual analogue scale $(0=$ most favourable, $10=$ least favourable), the study by Sutherland $1996^{54}$ asked patients and their carers to complete a questionnaire at initial follow-up and final follow-up at eight weeks. The questions included convenience of performing clean non-coated self catheterisation, ease of handling the catheter, comfort of insertion, and overall opinion. A significant difference favouring the hydrophilic catheter was reported for convenience (mean difference -1.60 ( -3.48 to 0.28$), \mathrm{P}=0.09$ ) and comfort of insertion (mean difference -1.50 ( -3.21 to 0.21$), \mathrm{P}=0.09$ ), but there was little difference with respect to handling (mean difference $0.00(-1.81$ to 1.81$), \mathrm{P}=1.00$ ) and general opinion of the catheter (mean difference -0.60 ( -2.36 to 1.16$), \mathrm{P}=0.50$ ).
Pachler et al $1999^{53}$ reported that fewer patients using hydrophilic catheters had problems introducing the catheter (risk ratio 0.50 ( 0.05 to 5.24$), \mathrm{P}=0.56)$, but a greater number reported a burning sensation (risk ratio 2.00(0.19 to 20.97), $\mathrm{P}=0.56)$ and pain (risk ratio 1.50 (0.27 to 8.38$), \mathrm{P}=0.64$ ) when introducing the catheter. An equal number reported a burning sensation or pain after catheter removal (risk ratio $1.00(0.15$ to 6.67), $\mathrm{P}=1.00$ ). None of the results was statistically significant. Giannantoini et al $2001^{27}$ also measured patient satisfaction associated with gel reservoir catheters using a visual analogue scale. The areas included in this measure were learning, inserting, extracting, comfort, and handling ease. Overall, use of gel reservoir catheters was associated with significantly greater patient satisfaction compared with use of non-coated catheters (mean difference in total score 2.39 (1.29 to 3.49), $\mathrm{P}=0.0001)$.

Neither of the studies comparing multiple use of non-coated catheters with single use of sterile non-coated catheters included a measure of patient preference or comfort.

\section{Number of catheters used per day or week}

One study reported frequency of catheterisation. Duffy et al $1995^{26}$ reported that people using multiple use non-coated catheters catheterised an average of three times per day, while those using sterile (single use) non-coated catheters catheterised 2.8 times per day (mean difference 0.20 ( -0.28 to 0.68 ), $\mathrm{P}=0.42$ ).

\section{Cost effectiveness}

The results of the cost effectiveness analysis indicate that, over a lifetime, gel reservoir catheters are $£ 28369$ more costly and result in an average gain of 0.522 QALYs per patient compared with clean non-coated catheters. This yields an ICER of $£ 54$ 350 per QALY gained, which exceeds the $£ 20000$ to $£ 30000$ threshold considered cost effective by UK policy makers. According to the results of the model, hydrophilic catheters are less effective than gel reservoir and their cost per QALY gained compared with clean non-coated catheters (changed once weekly) is higher. They are therefore deemed not cost effective because of extended dominance. Clean non-coated catheters are the next most effective method of catheterisation and are the most cost effective method of intermittent self catheterisation in $89.2 \%$ of model iterations. Compared with clean non-coated catheters changed once weekly, clean non-coated catheters changed once daily and sterile non-coated catheters changed once per use are less effective and more expensive; they are therefore not cost effective due to dominance.

If clean non-coated catheters are not considered a viable option for intermittent self catheterisation, gel reservoir catheters are the most cost effective. Compared with hydrophilic catheters, they cost an extra $£ 1373$ and result in a gain of 0.446 QALYs, giving an ICER of $£ 3075$ per QALY gained. Compared with hydrophilic catheters, gel reservoir catheters are cost effective in $84.6 \%$ of model iterations. The results of the cost effectiveness analysis are presented in fig $3 \Downarrow$ and table $6 \Downarrow$.

\section{Scenario and sensitivity analyses Urethral complications}

When the relative risk of urethral complications associated with each type of coated catheter is reduced to zero and the cost of complications is doubled (that is, hydrophilic catheters prevent $100 \%$ of urethral complications and those that occur with the use of other catheter types are twice as expensive as assumed 
in the base case), the conclusion of the analysis is unchanged. This is true regardless of whether multiple use non-coated catheters are considered an option.

\section{Antimicrobial resistance}

The conclusions of the model were robust to simultaneously varying the probability of the risk of treatment failure and multidrug resistant UTI to the upper limit of each input's 95\% confidence interval. This shows that, given current understanding of the scope of antibiotic resistance, multiple use non-coated catheters are the most cost effective option for intermittent self catheterisation.

\section{Baseline risk of UTI}

Clean non-coated catheterisation (one per week) remained the most cost effective strategy when the baseline risk of UTI was varied in threshold analysis between 1.4 and 3.0 (annual probability of $68 \%$ to $95 \%$ ).

\section{Number of non-coated catheters used}

The number of clean non-coated catheters used per year was varied between an average of 60 per year (average 5 per month) and 1825 per year (average 5 per day) in a threshold analysis. Assuming the same relative effectiveness as reported by Duffy $1995,{ }^{26}$ clean non-coated catheterisation ceases to be the most cost effective option when an average of 738 non-coated catheters is used per year (equivalent to about 62 catheters per month or 2 per day assuming consistent use).

\section{Intermittent self catheterisation in patients with bladder dysfunction not due to spinal cord injury}

A separate set of probabilities and utilities was collected in order to run a scenario analysis for patients with bladder dysfunction that is not caused by spinal cord injury. Assuming that each type of catheter exhibits the same relative efficacy in this population, the conclusion of this scenario analysis is the same as that for patients with spinal cord injury: clean non-coated catheterisation is the most cost effective method of intermittent self catheterisation (gel reservoir catheters are associated with a cost of $£ 149559$ per QALY gained); when clean non-coated catheterisation is not an option, gel reservoir catheters represent the most cost effective alternative. In both cases, sterile non-coated catheterisation is excluded from the analysis by dominance and hydrophilic catheters by extended dominance. However, the usefulness of this analysis is limited because the data used to inform estimates of clinical effectiveness was conducted primarily in men with spinal cord injury.

\section{Discussion}

The results of our systematic review show that use of different types of catheter for intermittent self catheterisation is associated with slightly different rates of symptomatic UTI. Although some of these differences are statistically significant, all are associated with wide and overlapping confidence intervals, conferring uncertainty as to whether the effects are of clinical significance. Our cost utility model was constructed to take this uncertainty into account. The results of the model show that, although gel reservoir catheters are the most effective type for intermittent self catheterisation, they are associated with a cost of over $£ 50$ 000 per QALY. Therefore, they are not considered cost effective in an NHS context. ${ }^{14}$ At a cost effectiveness threshold of $£ 20$ 000-30 000 per QALY, clean non-coated catheterisation is the most cost effective method of intermittent self catheterisation in $100 \%$ of model simulations. If clean non-coated catheters are not considered a relevant option, gel reservoir catheters are slightly more effective than hydrophilic with a small additional cost and an incremental cost effectiveness ratio of about $£ 3000$ per QALY.

\section{Strengths and limitations of study}

This conclusion was robust to a wide range of sensitivity analyses, including an increased probability of urethral complications that may be associated with the use of non-coated catheters. This is largely due to the magnitude of difference in resource use between sterile and clean methods of catheterisation. If the number of non-coated catheters used is varied in one-way sensitivity analysis, clean non-coated catheters cease to be the most cost effective choice when patients use an average of more than two per day. Compliance and behaviour are therefore important factors for healthcare workers to consider when prescribing an intermittent self catheterisation regimen. This analysis did not take into account the dynamic and extremely complex nature of antimicrobial resistance. Although the NICE guideline development group sought to use the most current, relevant estimates to inform this analysis, data about the prevalence of and mortality associated with antibiotic resistant UTI is limited, and it is impossible to predict the future of this phenomenon. If the prevalence and clinical and economic impacts of antimicrobial resistance increase beyond the extreme values used in this model, then the cost effectiveness of clean intermittent catheterisation in this population may have to be revisited.

As in most cost utility analyses, the current model assumes that an individual's quality of life is dependent on the health outcome achieved as a consequence of each intervention rather than factors related to the healthcare process itself. Where preferences have implications for costs and outcomes, it is important that cost effectiveness models incorporate this in their structure (such as by allowing for different compliance rates) and parameter values. However, it is difficult to find data to inform such estimates. The systematic review revealed that studies which reported a measure of patient preference or comfort used visual analogue scales and dichotomous questions that addressed slightly different outcomes. Therefore, although there was a significant preference for gel reservoir compared with non-coated catheters and conflicting results for hydrophilic catheters compared with non-coated, what this means in practice is unclear.

\section{Implications of results}

Individual patients may find one type of catheter more comfortable or easier to use than another and therefore derive a benefit that is not captured in the model. ${ }^{56}$ Currently, there is no consensus over the appropriate method to incorporate individual patient preference for a particular treatment that is not reflected in decisions made at the population level within the standard cost effectiveness approach. It has been suggested that one way to incorporate individual patient preference would be to adopt a two part decision process which gives the patient the choice of the most cost effective treatment plus all cheaper options. ${ }^{57}$ In other words, if patients have a strong preference for hydrophilic over gel reservoir catheters, an argument could be made for allowing them to choose this less costly option. It is important to note that under this rule patients would not be given a choice of therapies that are more costly than the most cost effective treatment, ${ }^{57}$ which do not represent value to users of the NHS as a whole. ${ }^{52}$ Therefore, this line of reasoning cannot be extended to patients who are able to use clean (multiple use) 
non-coated catheters but prefer not to, nor to patients who prefer sterile non-coated catheters to gel reservoir or hydrophilic catheters.

After consultation with registered guideline stakeholders (including manufacturers, NHS trusts, and patient groups), the guideline development group revisited the recommendation in light of issues raised regarding implementation and quality of the evidence base. There was concern that the designation of non-coated catheters as single use items would make clinicians liable for catheter associated infections caused by intermittent self catheterisation if they recommended clean non-coated catheterisation. There was also concern that advising patients to disregard the single use symbol on this device would lead to confusion and safety issues in other areas of care and that evidence as to the optimal method of cleaning non-coated catheters had not been included in our searches and was likely lacking from the evidence base. In addition, reusing a device labelled as single use may be considered similar to making an off-label recommendation, where robust clinical evidence is required. Given inadequate blinding, allocation concealment, and small sample size, the evidence on which this recommendation was based was considered to be of low to very low quality. Based on these concerns, the recommendation was amended before publication of the guideline. The recommendation states that patients should be given a choice between hydrophilic and gel reservoir catheters. For a comprehensive discussion of the considerations guiding this recommendation please see section 10.5.1.5 of the full guideline (http://guidance.nice.org.uk/CG139/Guidance/pdf/English). ${ }^{11}$

Currently, the uncertainty surrounding this question represents a potentially large opportunity cost for patients within the NHS. As a result of the issues raised by this systematic review and cost effectiveness model, this question has been identified as a priority for further research arising from the 2012 NICE infection prevention and control guideline. ${ }^{11}$ Ideally, a four or five arm randomised control trial is required to compare the relative effectiveness of each method of intermittent self catheterisation. The trial population should include a diverse population of people who regularly self catheterise, including wheelchair users, and the study should be of at least one year's duration. Primary outcome measures ought to include the incidence of symptomatic UTI and full spectrum of UTI associated complications (including bacteraemia, pylonephritis, and antibiotic resistant infection), mortality, patient comfort and preference, quality of life, clinical symptoms of urethral damage, and costs. High quality research into this area is urgently needed in order to ensure patients across the NHS are treated with high quality, cost effective care.

We thank Kathy Zebracki, Lawrence Vogel, Caroline Anderson, and Kathleen Chlan for providing us with access to SF-12 data collected from their research cohort. We thank Joanna Ashe for designing the search strategy and generating the bibliography and David Wonderling for his comments on the manuscript.

Contributors: All authors contributed to the protocol for this work. SH systematically reviewed, appraised, and analysed the included clinical studies, while SLB designed, built, and analysed the cost effectiveness model. SW, EH, and CP critically appraised all included clinical evidence and contributed to the design and interpretation of the cost effectiveness analysis. SLB wrote the first draft of the manuscript, which was subsequently edited by all authors, who have approved the final version. All authors had full access to the data (including statistical results and tables) and take responsibility for the integrity of the data and accuracy of the analysis. CP will act as guarantor.
Funding: This work was undertaken by the National Clinical Guideline Centre (SLB and $\mathrm{SH}$ ), which received funding from the National Institute for Health and Clinical Excellence (NICE). The views expressed in this publication are those of the authors and not necessarily those of the institute.

Competing interests: All authors have completed the ICMJE uniform disclosure form at www.icmje.org/coi_disclosure.pdf (available on request from the corresponding author) and declare: no support from any organisation for the submitted work; no financial relationships with any organisations that might have an interest in the submitted work in the previous three years; no other relationships or activities that could appear to have influenced the submitted work.

Ethical approval: Not required as the study used only publicly available data sources.

Data sharing: Technical appendix available at www.nice.org.uk/ nicemedia/live/13684/58655/58655.pdf.

1 Plowman R, Graves N, Esquivel J, Roberts JA. An economic model to assess the cost and benefits of the routine use of silver alloy coated urinary catheters to reduce the risk of urinary tract infections in catheterized patients. J Hosp Infect 2001;48:33-42.

2 Plowman R, Graves N, Griffin M, Roberts JA, Swan AV, Cookson B, et al. The socio-economic burden of hospital acquired infection: executive summary. Health Protection Agency; 1999.

3 Pilloni S, Krhut J, Mair D, Madersbacher H, Kessler TM. Intermittent catheterisation in older people: a valuable alternative to an indwelling catheter? Age Aging 2005;34:34-60.

4 Larsen LD, Chamberlin DA, Khonsari F, Ahlering TE. Retrospective analysis of urologic complications in male patients with spinal cord injury managed with and without indwelling urinary catheters. Urology 1997; 50:418-22.

5 Wyndaele JJ. Complications of intermittent catheterization: their prevention and treatment. Spinal Cord 2002;40:536-41.

6 Rainville NC. The current nursing procedure for intermittent urinary catheterisation in rehabilitation facilities. Rehabil Nurs 1994;19:330-3.

7 Buckley B, Grant A. What is the most effective method of neurogenic bladder dysfunction? BMJ 2009;338:827-8.

8 Moore KN, Burt J, Voaklander DC. Intermittent catheterization in the rehabilitation setting: a comparison of clean and sterile technique. Clin Rehabil 2006;20:461-8.

9 Moore KN, Fader M, Getliffe K. Long-term bladder management by intermittent catheterisation in adults and children. Cochrane Database Syst Rev 2007;(4):CD006008

10 Wyndaele JJ. Intermittent catheterisation: which is the optimal technique? Spinal Cord 2002;40:432-7.

11 National Clinical Guideline Centre. Infection: prevention and control of healthcare-asociated infections in primary and community care. Royal College of Physicians; 2012. Report No CG139.

12 Warren JW. Catheter-associated urinary tract infections. Infect Dis Clin North Am 1997;11:609-22.

13 National Clinical Guideline Centre. Appedices. In: Infection: prevention and control of healthcare-asociated infections in primary and community care. Royal College of Physicians; 2012. Report No CG139. http://guidance.nice.org.uk/CG139/Guidance/ Appendices/pdf/English

14 National Institute for Health and Clinical Excellence. The guidelines manual 2009. www. nice.org.uk/aboutnice/howwework/developingniceclinicalguidelines/ clinicalguidelinedevelopmentmethods/GuidelinesManual2009.jsp.

15 Cochrane IMS. GRADEpro. http://ims.cochrane.org/gradepro.

16 Review Manager (RevMan). Version 5.1. Nordic Cochrane Centre, Cochrane Collaboration, 2011. http://ims.cochrane.org/revman.

17 Briggs A, Sculpher M. An introduction to Markov modelling for economic evaluation. Pharmacoeconomics 1998;13:397-409.

18 National Spinal Cord Injury Statistical Centre. Facts and figures at a glance . 2011. https: //www.nscisc.uab.edu/

19 Office for National Statistics. England and Wales interim life tables 1980-82 to 2007-09. 2010. www.statistics.gov.uk/STATBASE/Product.asp?vink=14459.

20 Frankel HL, Coll JR, Charlifue SW, Whiteneck CG, Gardner BP, Krishnan KR, et al. Long-term survival in spinal cord injury: a fifty-year investigation. Spinal Cord 1998;36:266-74.

21 Bermingham SL, Ashe JF. A systematic review of the impact of urinary tract infection on health related quality of life. BJU Int 2012, doi:10.1111/j.1464-410X.2012.11337.x.

22 National Collaborating Centre for Women's and Children's Health. Urinary tract infection in children: diagnosis, treatment and long-term management. RCOG Press, 2007. Report No CG54.

23 Cranny G, Elliot R, Weatherly H, Chambers D, Hawkins N, Myers L, et al. A systematic review and economic model of switching from non-glycopeptide to glycopeptide antibiotic prophylaxis for surgery. Health Technol Assess 2008;12:35-61.

24 Cardenas DD, Hoffman JM. Hydrophilic catheters versus noncoated catheters for reducing the incidence of urinary tract infections: a randomized controlled trial. Arch Phys Med Rehabil 2009;90:1668-71.

25 De Ridder DJ, Everaert K, Fernandez LG, Valero JV, Duran AB, Abrisqueta ML, et al. Intermittent catheterisation with hydrophilic-coated catheters (SpeediCath) reduces the risk of clinical urinary tract infection in spinal cord injured patients: a prospective risk of clinical urinary tract infection in spinal cord injured patients:
randomised parallel comparative trial. Eur Urol 2005;48:991-5.

26 Duffy LM, Cleary J, Ahern S, Kuskowski MA, West M, Wheeler L, et al. Clean intermittent catheterization: safe, cost-effective bladder management for male residents of VA nursing homes. J Am Geriatr Soc 1995;43:865-70.

27 Giannantoni A, Di Stasi SM, Scivoletto G, Virgili G, Dolci S, Porena M. Intermittent catheterization with a prelubricated catheter in spinal cord injured patients: a prospective randomized crossover study. J Urol 2001;166:130-3. 


\section{What is already known on this subject}

Catheter associated urinary tract infection is a leading cause of morbidity and mortality in people using urinary catheters Because the risk of infection is greatest with indwelling catheters, intermittent catheterisation is the preferred option for bladder management The effectiveness and cost effectiveness of the different materials and methods of intermittent self catheterisation have never been systematically compared

\section{What this study adds}

Best available evidence indicates that the type of intermittent catheter used for intermittent self catheterisation has little effect on the rate of symptomatic infection and a large impact on cost

Clean non-coated catheterisation is the most cost effective method of intermittent self catheterisation; where this is not a viable option, gel reservoir catheters may be more cost effective than hydrophilic catheters

The current evidence base is limited, and additional data about the incidence of infection, urethral complications, patient compliance, methods of cleaning catheters, and quality of life are needed before such a change in practice is implemented

28 King RB, Carlson CE, Mervine J, Wu Y, Yarkony GM. Clean and sterile intermittent catheterization methods in hospitalized patients with spinal cord injury. Arch Phys Med Rehabil 1992;73:798-802.

29 Dow G, Rao P, Harding G, Brunka J, Kennedy J, Alfa M, et al. A prospective, randomized trial of 3 or 4 days of ciprofloxacin treatment for acute urinary tract infection in patients with spinal cord injury. Clin Infect Dis 2004;39:658-64.

30 Waites KB, Chen Y, DeVivo MJ, Cancupp KC, Moser SA. Antimicrobial resistance in gram-negative bacteria isolated from the urinary tract in community-residing persons with spinal cord injury. Arch Phys Med Rehabil 2000;81:764-9.

31 Mylotte J, Kahler L, Grahm R, Young L, Goodenough S. Prospective surveillance for antibiotic-resistant organisma in patients with spinal cord injury admitted to an acute rehabilitation unit. Am J Infect Control 2000;28:291-7.

32 Saint S. Clinical and economic consequences of nosocomial catheter-related bacteriuria. Am J Infect Control 2000;28:68-75.

33 Klevens RM, Edwards JR, Gaynes RP. The impact of antimicrobial-resistant health care-associated infections on mortality in the United States. Clin Infect Dis 2008;47:927-30.

34 Montgomerie JZ, Chan E, Gilmore DS, Canawati HN, Sapico FL. Low mortality among patients with spinal cord injury in bacteremia. Rev Infect Dis 2011;13:867-71.

35 Stensballe J, Looms D, Nielsen PN, Tvede M. Hydrophilic-coated catheters for intermittent catheterisation reduce urethral micro trauma: a prospective, randomised, participant-blinded, crossover study of three different types of catheters. Eur Urol 2005;48:978-83.

36 Vaidyanathan S, Soni BM, Dundas S, Krishnan KR. Urethral cytology in spinal cord injury patients performing intermittent catheterisation. Paraplegia 2010;32:493-500.

37 Perrouin-Verbe B, Labat JJ, Richard I, Mauduyt de la Greve I, Buzelin JM, Mathe JF. Clean intermittent catheterisation from the acute period in spinal cord injury patients. Long term evaluation of urethral and genital tolerance. Paraplegia 1995;33:619-24.

38 Haran MJ, Lee BB, King MT, Marial O, Stockler MR. Health status rated with the Medical Outcomes Study 36-Item Short-Form Health Survey after spinal cord injury. Arch Phys Med Rehabil 2005;86:2290-5.

39 Lee BB, King MT, Simpson JM, Haran MJ, Stockler MR, Marial O, et al. Validity, responsiveness, and minimal important difference for the SF-6D health utility scale in a spinal cord injured population. Value Health 2008;11:680-8.

40 Vogel LC, Krajci KA, Anderson CJ. Adults with pediatric-onset spinal cord injuries: part 3: impact of medical complications. J Spinal Cord Med 2002;25:297-305.

41 Zebracki K, Anderson C, Chlan K, Vogel LC. Outcomes of adults with pediatric-onset spinal cord injury: Iongitudinal findings and implications on transition to adulthood. Topic Spinal Cord Inj Rehabil 2010;16:17-25.

42 Gray A, Rivero-Arias O, Clarke P. Estimating the association between SF-12 responses and EQ-5D utility values by response mapping. Med Decis Making 2006;26:18-29.

43 Wong SS, Narahari R, O'Riordan A, Pickard R. Simple urethral dilatation, endoscopic urethrotomy, and urethroplasty for urethral stricture disease in adult men. Cochrane Database Syst Rev 2010;(4):CD006934.
44 Cost Effectivness Analysis Registry. 2011. https://research.tufts-nemc.org/cear4/.

45 NHS Business Services Authority, NHS Prescription Services. Electronic drug tariff, October 2010. www.ppa.org.uk/edt/October_2010/mindex.htm.

46 Woodbury MG, Hayes KC, Askes HK. Intermittent catheterization practices following spinal cord injury: a national survey. Can J Urol 2008;15:4065-71.

47 Turner D, Little P, Raftery J, Turner S, Smith H, Rumsby K, et al. Cost effectiveness of management strategies for urinary tract infections: results from randomised controlled trial. BMJ 2010;340:c346.

48 Health Protection Agency. Diagnosis of UTI: quick reference guide for primary care 2010. www.hpa.org.uk/web/HPAwebFile/HPAweb_C/1194947404720.

49 Curtis L. The costs of health and social care 2010. Personal Social Services Research Unit, 2010.

50 Department of Health. NHS reference costs 2009-10. 2011. www.dh.gov.uk/en/ Publicationsandstatistics/Publications/PublicationsPolicyAndGuidance/DH_123459.

51 Doubliet $P$, Begg CB, Weinstein MC, Braun P, McNeil BJ. Probabilistic sensitivity analysis using Monte Carlo simulation. A practical approach. Med Decis Making 1985;5:157-77.

52 National Institute of Clinical Excellence. Social value judgements: principles for the development of NICE guidance . NICE, 2008. www.nice.org.uk/aboutnice/howwework/ socialvaluejudgements.jsp.

53 Pachler J, Frimodt-Moller C. A comparison of prelubricated hydrophilic and non-hydrophilic polyvinyl chloride catheters for urethral catheterization. BJU Int 1999;83:767-9.

54 Sutherland RS, Kogan BA, Baskin LS, Mevorach RA. Clean intermittent catheterization in boys using the LoFric catheter. J Urol 1996;156:2041-3.

55 Vapnek JM, Maynard FM, Kim J. A prospective randomized trial of the LoFric hydrophilic coated catheter versus conventional plastic catheter for clean intermittent catheterization. $J$ Urol 2003:169:994-8.

56 Diokno AC, Mitchell BA, Nash AJ, Kimbrough JA. Patient satisfaction and the LoFric catheter for clean intermittent catheterisation. J Urol 1995;153:349-51.

57 Dixon S. Including patient choice in cost effectiveness decision rules. Health Economics and Decision Science (HEDS) Discussion Paper 07/06. 2007. http://eprints. whiterose.ac. uk/10913/1/HEDS DP 07-06.pdf.

Accepted: 10 December 2012

\section{Cite this as: BMJ 2012;345:e8639}

This is an open-access article distributed under the terms of the Creative Commons Attribution Non-commercial License, which permits use, distribution, and reproduction in any medium, provided the original work is properly cited, the use is non commercial and is otherwise in compliance with the license. See: http://creativecommons.org/licenses/by$\mathrm{nc} / 2.0 /$ and http://creativecommons.org/licenses/by-nc/2.0/legalcode. 


\section{Tables}

Table 1| Baseline probabilities* of transition to key health states among individuals with spinal cord injury undertaking intermittent self catheterisation

\begin{tabular}{|c|c|c|}
\hline Transition to health state (based on fig 1 ) & $\begin{array}{c}\text { Mean }(95 \% \mathrm{Cl}) \text { probability of transition annually } \\
(\%)\end{array}$ & Data source \\
\hline 1. Symptomatic UTI & 67.7 (60.9 to 74.2$)$ & $\begin{array}{c}\text { Cardenas } 2009,{ }^{24} \text { De Ridder } 2005,{ }^{25} \text { Duffy } 1995,{ }^{26} \\
\text { Giannantoni } 2001,{ }^{27} \text { King } 1992^{28}\end{array}$ \\
\hline 2. UTI resistant to first line antibiotic & $8.3(0.0$ to 23.2$)$ & Dow 2004, ${ }^{29}$ Waites 2000, ${ }^{30}$ Mylotte $2000^{31}$ \\
\hline 3. Multidrug resistant UTI & $7.0(5.1$ to 9.2$)$ & Dow $2004,{ }^{29}$ Waites $2000,{ }^{30}$ Mylotte $2000^{31}$ \\
\hline 4. UTI associated bacteraemia & $3.6(3.4$ to 3.8$)$ & Saint $2000^{32}$ \\
\hline 5 Mortality due to multidrug resistant UTI & $2.6(1.3$ to 5.1$)$ & Klevens $2008^{33}$ \\
\hline 6. Mortality due to UTI associated bacteraemia & 7.7 (2.9 to 19.2$)$ & Montgomerie $2011^{34}$ \\
\hline 7. Urethral complications & $2.4(0.9$ to 6.2$)$ & Perrouin-Verbe $1995^{37}$ \\
\hline
\end{tabular}

UTI=urinary tract infection.

*Baseline probabilities based on using sterile non-coated catheters for intermittent self catheterisation. 
Table 2| Measures quality of life on EQ-5D instrument associated with key health states among individuals with spinal cord injury undertaking intermittent self catheterisation

\begin{tabular}{llc} 
Health state & Mean value $(95 \%$ Cl) & Data source \\
\hline No symptomatic UTI & $0.831(0.809$ to 0.852$)$ & Vogel $2002,{ }^{40}$ Zebracki $2010^{41}$ \\
\hline Symptomatic UTI & $0.782(0.764$ to 0.799$)$ & Vogel 2002, ${ }^{40}$ Zebracki $2010^{41}$ \\
\hline UTI resistant to first line antibiotics & $0.760(0.685$ to 0.834$)$ & Expert opinion \\
\hline Multidrug resistant UTI & $0.738(0.688$ to 0.787$)$ & Vogel $2002,{ }^{40}$ Zebracki $2010^{41}$ \\
\hline Catheter associated bacteraemia & $0.716(0.645$ to 0.786$)$ & Expert opinion \\
\hline Urethral complications & $0.738(0.688$ to 0.787$)$ & Assumed to be equal to that of multidrug resistant UTI \\
\hline & & \\
UTI=urinary tract infection. & & \\
\hline
\end{tabular}




\begin{tabular}{|c|c|}
\hline & Mean (range) values \\
\hline \multicolumn{2}{|l|}{ Costs of items (£) } \\
\hline Hydrophilic catheter (each) & $1.28(0.97-1.66)$ \\
\hline Gel reservoir catheter (each) & $1.36(0.98-1.43)$ \\
\hline Non-coated catheter (each) & $1.19(0.39-1.47)$ \\
\hline Lubricant for non-coated catheters (per sterile sachet) $\dagger$ & $0.24(0.21-0.26)$ \\
\hline Dispensing fee (per month) $\ddagger$ & $1.96(1.87-2.11)$ \\
\hline \multicolumn{2}{|l|}{ Number of items used per year } \\
\hline Sterile catheters & $1825(1460-2190)$ \\
\hline Clean non-coated catheters (using one per day) & $365(292-438)$ \\
\hline Clean non-coated catheters (using one per week) & $60(48-72)$ \\
\hline Sachets of lubricant† & $1825(1460-2190)$ \\
\hline \multicolumn{2}{|c|}{ Associated annual cost of each method of catheterisation (£) } \\
\hline Hydrophilic catheter & $2339(1657-3161)$ \\
\hline Gel reservoir catheter & $2482(1926-3127)$ \\
\hline Sterile non-coated catheter (one per use) & $4343(3109-5826)$ \\
\hline Clean non-coated catheter (one per day) & $864(710-1039)$ \\
\hline Clean non-coated catheter (one per week) & $502(411-606)$ \\
\hline
\end{tabular}

${ }^{*}$ Costs of items and numbers of clean non-coated catheters used annually derived from NHS Drug Tariff $2010,{ }^{45}$ numbers of sterile catheters used annually derived from Woodbury $2008 .^{46}$

†Assuming that $95 \%$ of people use water based lubricant at mean cost $£ 0.19$ (range $£ 0.18-0.19$ ) per $5 \mathrm{~g}$ sachet and $5 \%$ use lidocaine containing lubricant at mean cost $£ 1.20(£ 1.87-1.44)$ per $8.5 \mathrm{~g}$ sachet, ${ }^{45}$ and that lubricant is used for each catheterisation.

$\ddagger$ A dispensing fee was added to the cost of each month's worth of catheters and lubricant. Because gel reservoir and hydrophilic catheters do not require lubricant, the total monthly dispensing fee for these catheters was half that for non-coated catheters. 


\begin{tabular}{|c|c|c|}
\hline Health state and cost items & Mean (range) cost $(£)$ & Data sources \\
\hline \multicolumn{3}{|l|}{ Symptomatic UTI } \\
\hline Healthcare consultation* & $29.62(25.17-34.58)$ & PSSRU $2010,{ }^{49} \mathrm{NHS}$ reference costs $2011^{50}$ \\
\hline Dipstick analysis & $0.07(0.06-0.08)$ & NHS Drug Tariff $2010^{45}$ \\
\hline First line antibiotic treatment $†$ & $2.00(0.75-4.05)$ & NHS Drug Tariff $2010^{45}$ \\
\hline Dispensing fee & $1.96(1.87-2.11)$ & NHS Drug Tariff $2010^{45}$ \\
\hline Additional catheters & Varies depending on catheter & NHS Drug Tariff $2010^{45}$ \\
\hline \multicolumn{3}{|l|}{ Infection resistant to first line antibiotics } \\
\hline Healthcare consultation ${ }^{*}$ & $29.62(25.17-34.58)$ & PSSRU $2010,{ }^{49} \mathrm{NHS}$ reference costs $2011^{50}$ \\
\hline Urine analysis & $7.00(5.00-9.00)$ & NHS reference costs $2011^{50}$ \\
\hline Second line antibiotic treatment $\ddagger$ & $13.48(2.33-50.40)$ & NHS Drug Tariff $2010^{45}$ \\
\hline Dispensing fee & $1.96(1.87-2.11)$ & NHS Drug Tariff $2010^{45}$ \\
\hline Additional catheters & Varies depending on catheter & NHS Drug Tariff $2010^{45}$ \\
\hline \multicolumn{3}{|l|}{ Multidrug resistant UTI } \\
\hline Healthcare consultation ${ }^{*}$ & $29.62(25.17-34.58)$ & PSSRU $2010,{ }^{49} \mathrm{NHS}$ reference costs $2011^{50}$ \\
\hline Urine analysis & $7.00(5.00-9.00)$ & NHS reference costs $2011^{50}$ \\
\hline $\begin{array}{l}\text { Non-elective inpatient admission (HRG episodes LA04E } \\
\text { and LA04F)§ }\end{array}$ & $2123(1394-3057)$ & NHS reference costs $2011^{50}$ \\
\hline \multicolumn{3}{|l|}{ Bacteraemia } \\
\hline Healthcare consultation $^{*}$ & $29.62(25.17-34.58)$ & PSSRU $2010,{ }^{49} \mathrm{NHS}$ reference costs $2011^{50}$ \\
\hline Urine analysis & $7.00(5.00-9.00)$ & NHS reference costs $2011^{50}$ \\
\hline Blood test & $7.00(5.00-9.00)$ & NHS reference costs $2011^{50}$ \\
\hline $\begin{array}{l}\text { Non-elective inpatient admission (HRG episode } \\
\text { LA04D) }\end{array}$ & 3155 (1798-4897) & NHS reference costs $2011^{50}$ \\
\hline \multicolumn{3}{|l|}{ Urethral complications } \\
\hline Urethral procedure $^{\star \star}$ & $1268(908-1399)$ & NHS reference costs $2011^{50}$ \\
\hline \multicolumn{3}{|c|}{ Associated cost of treating each type of catheter associated UTI } \\
\hline Symptomatic UTI & $49(44-55)$ & - \\
\hline First-line antibiotic resistant UTI & $68(52-96)$ & - \\
\hline Multidrug resistant UTI & $2160(1430-3095)$ & 一 \\
\hline Bacteraemia & $3191(1834-4934)$ & - \\
\hline Urethral complications & $1267(745-1919)$ & - \\
\hline
\end{tabular}

\section{$\mathrm{UTI}=$ urinary tract infection. PSSRU = Personal Social Services Research Unit. HRG = Healthcare Resource Group.}

*Assuming that the healthcare provider usually consulted was a GP (in $80 \%$ of cases), followed by community nurse specialist (10\%) and hospital emergency department (10\%). Costs of GP and community nurse consultations obtained from PSSRU ${ }^{49}$ and cost of emergency care from NHS reference costs. ${ }^{50}$

†In England and Wales first line treatment for symptomatic UTI typically includes the antibiotics trimethoprim (200 mg twice daily for 5 days), nitrofurantoin (50 mg 4 times daily for 5 days), cefalexin ( $500 \mathrm{mg}$ twice daily for 5 days), and pivmecillinam (200 $\mathrm{mg} 3$ times daily for 3 days), with prescribing varied between regions and practices. The point estimate was calculated as a simple mean of the cost of a course of each drug as listed by NHS Drug Tariff, ${ }^{45}$ with the upper and lower range informed by the most and least expensive treatment options.

‡Cost of second line antibiotics was calculated as a simple mean of the cost of a course of each drug as listed in the NHS Drug Tariff, ${ }^{45}$ with the upper and lower range informed by the most and least expensive treatment options: ciprofloxacin (250 mg 3 times daily for 7 days); cefaclor ( $250 \mathrm{mg} 3$ times daily for 7 days); cefixime (200 mg once daily for 7 days); norfloxacin (400 mg twice daily for 7 days); ofloxacin (400 mg once daily for 7 days); pivmecillinam (400 mg 4 times daily for 7 days).

§Patients with multidrug resistant UTI are usually admitted to hospital for intravenous drug therapy. The cost of treatment was calculated as a weighted average reference cost for kidney infection or UTI with intermediate complications (HRG episode LA04E) or without complications (HRG episode LA04F). Costs included excess bed days and were weighted according to reported activity with $73 \%$ of total cost attributed to LA04E.

ףThe cost of treatment for UTI associated bacteraemia was assumed to be equal to the non-elective reference cost for kidney infection or UTI with major complications (HRG episode LA04D). In the UK, bacteraemia caused by resistant organisms does not seem to have a substantial impact on length of hospital stay compared with bacteraemia caused by susceptible organisms.

${ }^{\star \star}$ The cost of treating a urethral complication was estimated based on the NHS reference costs ${ }^{50}$ of urethral disorders and intermediate or minor procedures without complications (HRG episode LB30B). 


\begin{tabular}{|c|c|c|c|c|c|c|}
\hline Study & Trial design and population & No of patients & $\begin{array}{l}\text { Mean age } \\
\text { (years) }\end{array}$ & $\%$ male & Follow-up period & Outcomes reported \\
\hline \multicolumn{7}{|c|}{ Hydrophilic catheters $v$ sterile non-coated catheters (one per use) } \\
\hline Vapnek $2003^{55}$ & RCT of men with neurogenic bladders & 62 & 40 & $100 \%$ & 1 year & Mean monthly UTI \\
\hline Cardenas $2009^{24}$ & RCT of patients with spinal cord injury & 56 & 41 & $52 \%$ & 1 year & $\begin{array}{c}\text { Patients with } \geq 1 \text { UTI. Total } \\
\text { UTIs at } 1 \text { year }\end{array}$ \\
\hline De Ridder $2005^{25}$ & $\begin{array}{l}\mathrm{RCT} \text { of men with spinal cord injury } \\
\text { presenting with neurogenic bladder and } \\
\text { sphincter disorders }\end{array}$ & 123 & 37 & $100 \%$ & 1 year & $\begin{array}{l}\text { Patients with } \geq 1 \text { UTI. Patient } \\
\text { or carer satisfaction }\end{array}$ \\
\hline Sutherland $1996^{54}$ & $\begin{array}{c}\text { RCT of men with neurogenic bladder due } \\
\text { to spinal cord injury, Hinman syndrome, } \\
\text { or spinal dysraphism }\end{array}$ & 33 & 12 & $100 \%$ & 8 weeks & Patient or carer satisfaction \\
\hline Pachler $1999^{53}$ & $\begin{array}{l}\text { RCoT of men with urinary retention } \\
\text { caused by prostatic enlargement }\end{array}$ & 43 & 71 & $100 \%$ & 3 weeks in each arm & $\begin{array}{l}\text { Problems, burning, and pain } \\
\text { at introduction and removal of } \\
\text { catheter }\end{array}$ \\
\hline \multicolumn{7}{|c|}{ Gel reservoir catheters $v$ sterile non-coated catheters (one per use) } \\
\hline Giannantoni $2001^{27}$ & $\begin{array}{l}\text { RCoT of patients with neurogenic } \\
\text { bladder due to recent spinal cord injury }\end{array}$ & 18 & 38 & $89 \%$ & 7 weeks in each arm & $\begin{array}{c}\text { Patients with } \geq 1 \text { UTI. Patient } \\
\text { comfort }\end{array}$ \\
\hline \multicolumn{7}{|c|}{ Clean non-coated catheters (one per day) $v$ sterile non-coated catheters (one per use) } \\
\hline King $1992^{28}$ & $\begin{array}{l}\mathrm{RCT} \text { of people with spinal cord injury in } \\
\text { inpatient rehabilitation programmes } \\
\text { requiring intermittent self catheterisation }\end{array}$ & 46 & 29 & $87 \%$ & $\begin{array}{l}28 \text { days or until } \\
\text { infection }\end{array}$ & Patients with $\geq 1$ UTI \\
\hline \multicolumn{7}{|c|}{ Clean non coated catheters (one per week) $v$ sterile non-coated catheters (one per use) } \\
\hline Duffy $1995^{26}$ & $\begin{array}{c}\text { RCT of male residents of long term care } \\
\text { homes requiring intermittent self } \\
\text { catheterisation }\end{array}$ & 80 & 72 & $100 \%$ & $\begin{array}{c}\text { Mean } 64 \text { days (range } \\
15-107)\end{array}$ & $\begin{array}{l}\text { Patients with } \geq 1 \text { UTI. No of } \\
\text { catheterisations per day }\end{array}$ \\
\hline
\end{tabular}

$\mathrm{RCT}=$ randomised controlled trial. $\mathrm{RCOT}=$ randomised crossover trial. UTI = urinary tract infection. 
Table $6 \mid$ Results of base case analysis (probabilistic) of comparative costs, health gains, and cost effectiveness of different catheters and methods for performing intermittent self catheterisation in the community

\begin{tabular}{|c|c|c|c|c|c|c|c|c|c|c|}
\hline \multirow[b]{3}{*}{ Catheter } & \multicolumn{2}{|c|}{ Total values } & \multicolumn{2}{|c|}{ Incremental values } & \multicolumn{3}{|c|}{ Including clean non-coated catheters } & \multicolumn{3}{|c|}{ Excluding clean non-coated catheters } \\
\hline & \multirow[b]{2}{*}{ Cost $(\Sigma)$} & \multirow[b]{2}{*}{ QALYs } & \multirow[b]{2}{*}{$\begin{array}{l}\text { Cost } \\
(\Sigma)^{\star}\end{array}$} & \multirow[b]{2}{*}{ QALYs* } & \multirow{2}{*}{$\begin{array}{c}\text { Incremental } \\
\text { net benefit } \\
(£)\end{array}$} & \multicolumn{2}{|c|}{$\begin{array}{c}\text { Probability cost effective } \\
(\%)\end{array}$} & \multirow{2}{*}{$\begin{array}{l}\text { Incremental } \\
\text { net benefit } \\
\text { (£) }\end{array}$} & \multicolumn{2}{|c|}{$\begin{array}{l}\text { Probability cost effective } \\
(\%)\end{array}$} \\
\hline & & & & & & $\begin{array}{l}\text { At } £ 20000 \\
\text { threshold }\end{array}$ & $\begin{array}{l}\text { At } £ 30000 \\
\text { threshold }\end{array}$ & & $\begin{array}{l}\text { At } £ 20000 \\
\text { threshold }\end{array}$ & $\begin{array}{l}\text { At } £ 30000 \\
\text { threshold }\end{array}$ \\
\hline $\begin{array}{l}\text { Clean } \\
\text { non-coated } \\
\text { (one per } \\
\text { week) }\end{array}$ & 11879 & 11.928 & Baseline & Baseline & Baseline & 89.2 & 79.9 & - & - & - \\
\hline $\begin{array}{l}\text { Clean } \\
\text { non-coated } \\
\text { (one per day) }\end{array}$ & 17741 & 11.780 & 5862 & -0.148 & -8820 & 10.6 & 14.9 & - & - & - \\
\hline Hydrophilic & 38875 & 12.003 & 26997 & 0.076 & -16665 & 0 & 0 & Baseline & 14.7 & 8.9 \\
\hline Gel reservoir & 40248 & 12.450 & 28369 & 0.522 & 7556 & 0.2 & 5.6 & 7556 & 84.6 & 90.8 \\
\hline $\begin{array}{l}\text { Sterile } \\
\text { non-coated } \\
\text { (one per use) }\end{array}$ & 43594 & 11.880 & 31715 & -0.048 & -14747 & 0 & 0 & -14747 & 0.7 & 0.4 \\
\hline
\end{tabular}

QALYs = quality adjusted life years.

${ }^{*}$ Incremental costs and QALYs are calculated compared with the lowest cost option (clean non-coated catheters used once per week when multiple use of catheters is permitted; hydrophilic catheters when multiple use is not permitted). 


\section{Figures}

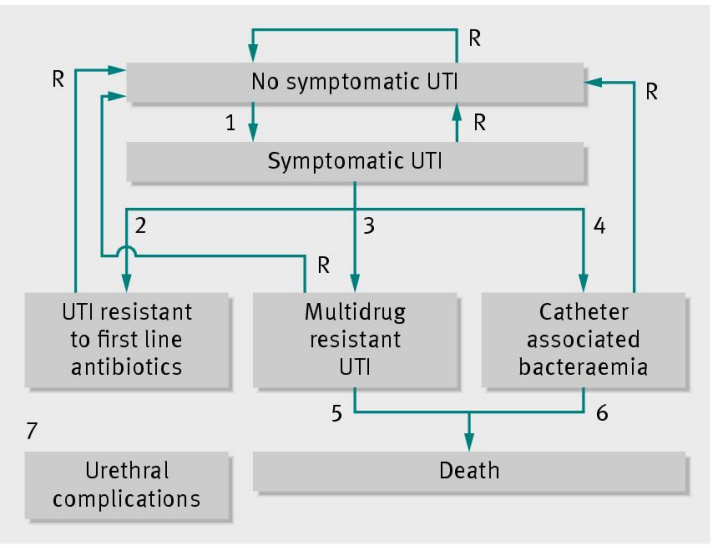

Definitions:

Symptomatic UTI = one or more symptom suggestive of UTI or self reported UTI requiring treatment, or both

UTI resistant to first line antibiotics $=$ patients with symptomatic relapse who require further antibiotic treatment within 28 days of the initial prescription Multidrug resistant UTI $=$ resistance to two or more classes of antimicrobial agents

Catheter associated bacteraemia $=$ presence of symptomatic UTI and the same organism to be isolated from the blood stream as in the urinary tract.

Death = Individuals in the model were exposed to a background rate of all

cause mortality for people with spinal cord injury. The transitions to death

cause mortalisy for people modh

than the background risk of death

than the background risk of death

$R=$ residual probability (that is, 1-(sum of all other transition probabilities

from that health state)

Fig 1 Schematic diagram of Markov model structure. The costs and consequences of catheter associated urinary tract infection (UTI) were modelled as movements between six health states of a Markov transition model

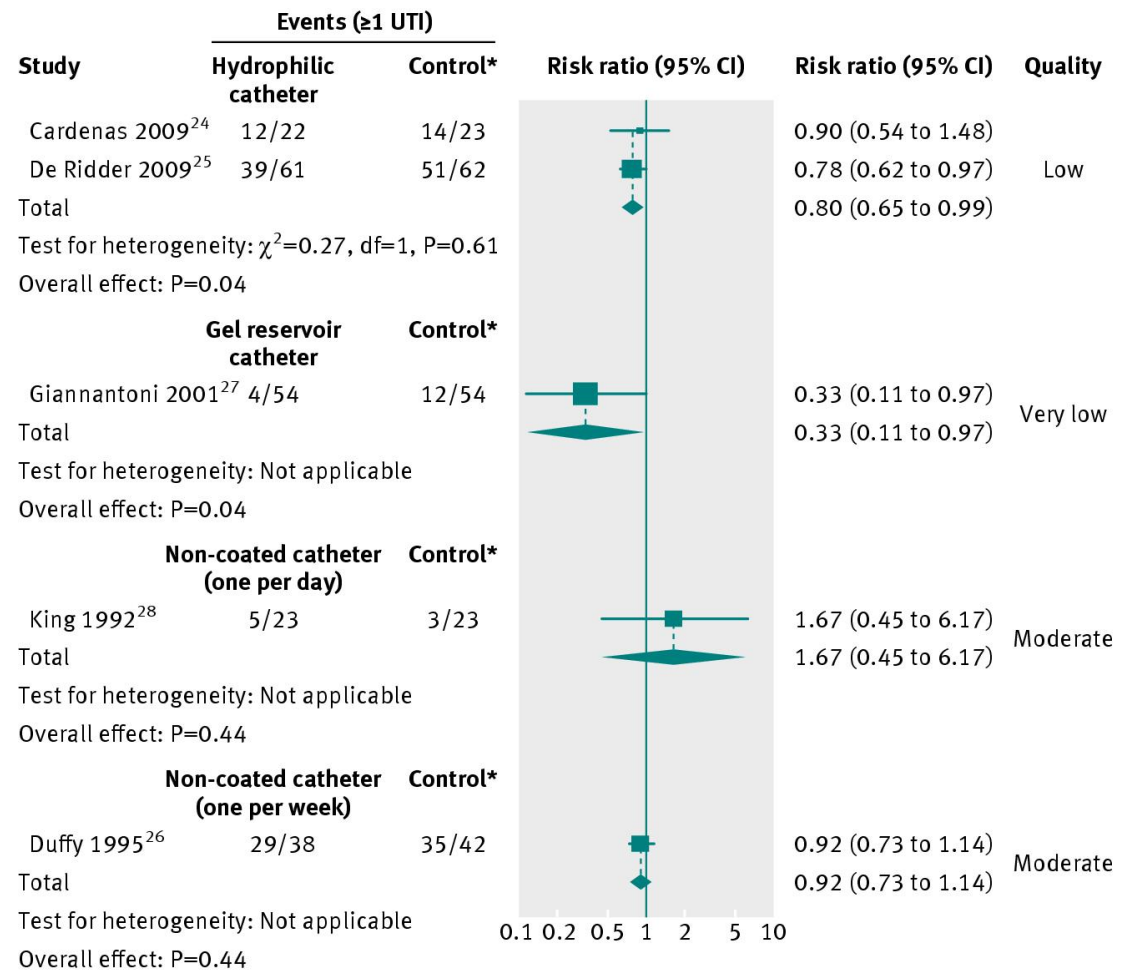

* Control $=$ sterile non-coated catheter (one per use)

Fig 2 Relative risks of adverse events ( $\geq 1$ urinary tract infection (UTI)) with different catheters and methods for performing intermittent self catheterisation in the community 
- Clean non-coated catheter (one per day)

O Clean non-coated catheter (one per week)

a Sterile non-coated catheter (one per use)

$\square$ Hydrophilic catheter

$\Delta$ Gel reservoir cathete

All comparators
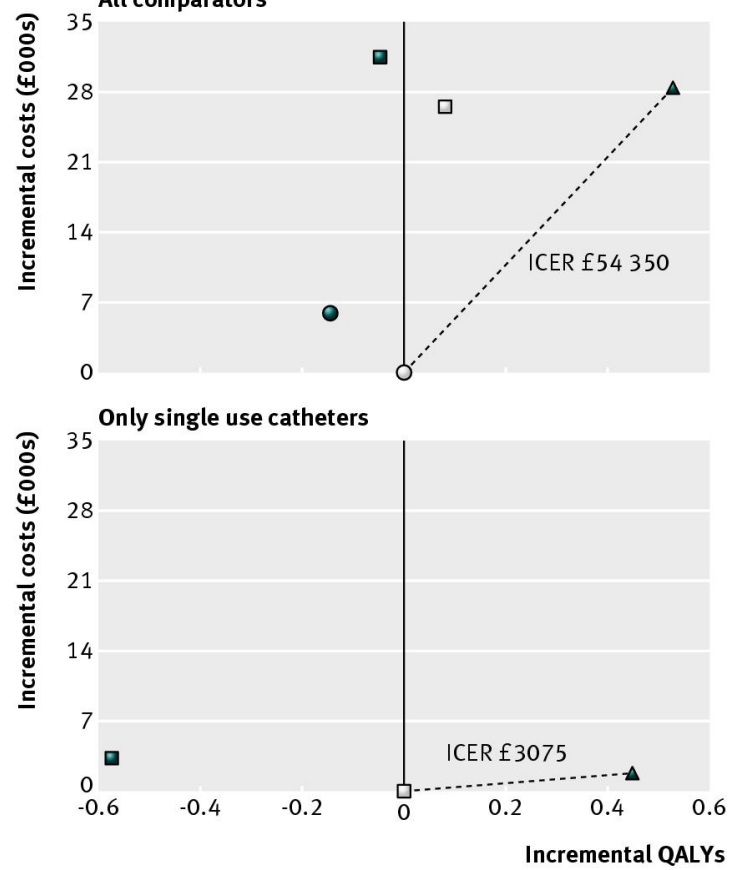

Fig 3 Results of base case analysis (probabilistic) of relative cost effectiveness of different catheters and methods for performing intermittent self catheterisation in the community. Costs and quality adjusted life years (QALYs) are means per person over a lifetime horizon. The dotted line represents the cost effectiveness frontier; the incremental cost effectiveness ratio (ICER) presented is for gel reservoir catheters compared with the least costly strategy in each scenario 